\title{
Transmission of M-type combined solitary wave in the birefringent fiber with comprehensive management
}

\author{
Yan XiaO ${ }^{1 *}$, XiaO-Qin Bai ${ }^{1}$, Chang-Yong Li ${ }^{2}$, Ting-Ting Lv ${ }^{1}$ \\ ${ }^{1}$ College of Physics and Electronics Engineering, Shanxi University, Taiyuan 030006, China \\ ${ }^{2}$ State Key Laboratory of Quantum Optics and Quantum Optic Devices, \\ Institute of Laser Spectroscopy, Shanxi University, Taiyuan, 030006, China \\ *Corresponding author: xiaoyan@sxu.edu.cn
}

\begin{abstract}
In this paper, we reveal the transmission properties of the M-type combined solitary wave in birefringent fiber with third-order dispersion, self-frequency shift, self-steepening, fifth-order nonlinearity and the gain (loss) effects. The numerical simulations show that the M-type solitary wave can be stably transmitted through 300 dispersion lengths via balancing the variety of effects. And it can even be stably transmitted under the condition of limited interference under a small perturbation of noise, amplitude and phase position. The results can provide certain references for the research of optical soliton communication and optical devices.
\end{abstract}

Keywords: M-type combined solitary wave, birefringent fiber, fifth-order nonlinearity.

\section{Introduction}

The optical soliton communication system becomes one of the hot research topics in recent years, since it has an extremely high rate and can also realize large capacity and long distance fiber communication [ $\underline{1}, \underline{2}]$. In 1966, KAO and HосKHAM []ㅡ firstly pointed out the possibility of transmitting information using optical fiber, thus laying the foundation of modern optical fiber communication and marking a significant milestone in optical fiber communication history. The optical soliton, which serves as a good candidate for optical communications in optical fiber, can keep orthoscopic transmission characteristics along the direction of propagation in a nonlinear optical device [4]. The transmission of bright soliton in optical fiber without distortion for the first time was observed experimentally in Bell Labs [్]. These features make the soliton become an excellent carrier of long distance transmission and high capacity information. The recent studies of an ultrashort optical pulse discover that the optical soliton communication can overcome the limit of the distance and speed in optical wave transmission system. 
A single mode fiber supports two degenerate modes that are polarized in two orthogonal directions. Under ideal cylindrical geometry and isotropic materials, a mode in one direction is not coupled to the mode in the orthogonal direction. However, small deviations from the cylindrical geometry or small fluctuations in material anisotropy lead to a mixture of the two mode in orthogonal directions and the mode degeneracy is broken. This property is referred to as modal birefringence [ $\underline{6}-\underline{8}]$. CHRISTODOULIDES and JOSEPH indicated that soliton pulses with orthogonal polarization component can keep unchanged waveform transmission in the birefringent fiber [9].

Within the classic scope, via the inverse scattering method, the bright soliton and the dark soliton were found to be the solutions of the nonlinear Schrödinger equation, which is the basic theoretical model of the picosecond light pulses transmission in optical fiber [10]. In the actual applications, however, the material dispersion, nonlinear effect and other effects existing in the optical fiber should be taken into account. Using the coupled nonlinear Schrödinger equation as the theoretical model of optical pulse transmission in birefringent fiber, the transmission properties and stability of optical soliton can be more specific described.

New types of combined chirp solitary wave solutions of high order Ginzburg-Landau equation with unsaturated absorption term were given by a suitable ansatz method, and the stabilities of the chirp solitary wave solutions were discussed by numerical simulation method [11]. In practical applications, the transmission mediums of optical pulse are asymmetrical due to the increase in transmission distance and the inaccuracy of craftsmanship, therefore more details of optical fiber must be prudent considered. Chirp bright and dark soliton solutions of the complex variable coefficient Ginzburg-Landau equation were calculated in 2006 [12].

In recent years, there have been many new studies on solitons. By means of the Hirota method, dark vector solitons of the coupled higher-order nonlinear Schrödinger equations are investigated in birefringent fiber or two-mode fiber [13]. Other research has found that the vector dark soliton solution for the coupled defocusing nonlinear Schrödinger equations is unstable to radiation modes with different diffraction coefficients [14]. A class of new spatiotemporal solitary solution to the nonlinear Schrödinger equation with a parabolic potential is reported analytically and numerically using the $F$-expansion method and homogeneous balance principle [15]. In consideration of higher-order nonlinear terms, an extended form of a simple equation method is proposed to construct exact soliton and solitary wave solutions of the higher-order nonlinear Schrödinger equation [16]. By adopting a complex amplitude ansatz solution which is composed of bright and dark solitary waves, the exact dipole soliton solution is investigated through an inhomogeneous cubic-quintic-septic medium [17].

In this article, we analyze the transmission characteristics of the M-type combined solitary wave in the birefringent fiber. Using the variable coefficients coupled nonlinear Schrödinger equation with the third-order dispersion, the self-frequency shift, the self-steepening, five-order nonlinear and the gain (loss) effect as the theoretical model, 
we simulated numerically the evolution characteristics of the M-type combined solitary wave and discussed its transmission stability when the small perturbation of noise, amplitude and phase position were considered respectively.

\section{Theoretical mode}

The two orthogonal modes of the single mode fiber have the same spatial distribution. The ideal fiber can keep strict cylindrical symmetry on the whole length and its two orthogonal modes are degenerate, which means that they have the same effective refractive index. In the actual applications, however, all the optical fibers will show a certain degree of mode birefringence for the unexpected changes in shape of the optical fiber or the influence of anisotropic stress. The birefringent fiber has two transmission shafts, which are the fast axis and the slow axis. The refractive index of the fast axis and the slow axis is different, and the light pulses pass through fibers at different speed along the two main shafts.

The coupled nonlinear Schrödinger equation can describe evolutions of the two polarization components of the optical fiber along the fast axis and the slow axis. Most of the time optical fibers we encountered are asymmetrical in the actual application, therefore we choose the variable coefficients coupled nonlinear Schrödinger equation as the theoretical model.

In the birefringent fiber, the basic theoretical model of transmission has the following form:

$$
\left\{\begin{array}{l}
i\left(\frac{\partial p}{\partial z}+\delta \frac{\partial p}{\partial t}\right)+\frac{1}{2} \beta_{2} \frac{\partial^{2} p}{\partial t^{2}}+\gamma_{3}\left(|p|^{2}+\frac{2}{3}|q|^{2}\right) p \\
\quad+i \beta_{3} \frac{\partial^{3} p}{\partial t^{3}}+i a_{3} \frac{\partial}{\partial t}\left(|p|^{2} p\right)+i a_{4} p \frac{\partial|p|^{2}}{\partial t}+a_{5}|p|^{4} p+i g p=0 \\
i\left(\frac{\partial q}{\partial z}-\delta \frac{\partial q}{\partial t}\right)+\frac{1}{2} \beta_{2} \frac{\partial^{2} q}{\partial t^{2}}+\gamma_{3}\left(|q|^{2}+\frac{2}{3}|p|^{2}\right) q \\
\quad+i \beta_{3} \frac{\partial^{3} q}{\partial t^{3}}+i a_{3} \frac{\partial}{\partial t}\left(|q|^{2} q\right)+i a_{4} q \frac{\partial|q|^{2}}{\partial t}+a_{5}|q|^{4} q+i g q=0
\end{array}\right.
$$

In the above equations, $p$ and $q$ are normalized amplitudes along the fast and slow axis, and they are all functions about $z$ and $t$, where $z$ represents the transmission distance and $t$ represents the delay time; $\delta$ represents the group velocity mismatch of the two polarized components; $\beta_{2}$ and $\beta_{3}$ represent the group velocity dispersion (GVD) and the third order dispersion (TOD), respectively; $\gamma_{3}$ represents the nonlinear effect; $a_{3}, a_{4}$ and $a_{5}$ represent the self-steepening, the self-frequency shift and five-order nonlinear effect, respectively; $g$ is the linear gain (loss). 
In the actual heterogeneous optical fiber, we assume that all the mentioned parameters have the following forms:

$$
\begin{aligned}
& \beta_{2}=b_{20}[1+a \sin (\sigma z)] \exp (\mu z) \\
& \beta_{3}=b_{30}[1+a \sin (\sigma z)] \exp (\mu z) \\
& \gamma_{3}=\gamma_{30}[1+a \sin (\sigma z)] \exp (\mu z) \\
& a_{3}=a_{30}[1+a \sin (\sigma z)] \exp (\mu z) \\
& a_{4}=a_{40}[1+a \sin (\sigma z)] \exp (\mu z) \\
& a_{5}=a_{50}[1+a \sin (\sigma z)] \exp (\mu z)
\end{aligned}
$$

In the above relationships $b_{20}, b_{30}, \gamma_{30}, a_{30}, a_{40}$, and $a_{50}$ are ideal optical fiber parameters; $a$ is a small constant, and it represents the volatility of optical parameters; $\sigma$ is the change in the optical fiber parameters cycle; $\mu$ is a small real constant.

\section{Numerically analysis and discussion}

All the parameters of Eq. (1), pulse and fiber should be determined before numerical simulation. The optical pulse passes through the fiber in the form of the combined solitary waves, as follows:

$$
\left\{\begin{array}{l}
p(0, t)=i \beta+A_{0} \tanh \left[\eta\left(t-T_{0}\right)\right] \operatorname{sech}\left[\eta\left(t-T_{0}\right)\right] \\
q(0, t)=i \beta+A_{0} \tanh \left[\eta\left(t-T_{0}\right)\right] \operatorname{sech}\left[\eta\left(t-T_{0}\right)\right]
\end{array}\right.
$$

where $\eta$ represents the reciprocal of pulse width, $T_{0}$ represents the center of the pulse, and we set $T_{0}=0 ; \beta$ and $A_{0}$ are real constants.

All the parameters of pulse and fiber are set as: $A_{0}=1.55, \beta=0.12, T_{0}=0, \eta=0.1$, $\delta=0.001, g=0.0001, \sigma=0.2, \mu=-0.08, a=0.1, b_{20}=0.4, b_{30}=0.08, \gamma_{30}=0.1$, $a_{30}=0.03, a_{40}=-0.02$, and $a_{50}=0.002$.

Then the transmission diagram of a M-type combined solitary wave can be obtained, as in Fig. 1. And we can see that transmission of soliton is based on the shape of the M. The M-type combined solitary wave can be stably transmitted through 300 dispersion lengths in the normal dispersion regime of birefringent fiber, except there is a self-adjusting process at the beginning. There are slight fluctuations on both sides of the M-type combined solitary wave. 


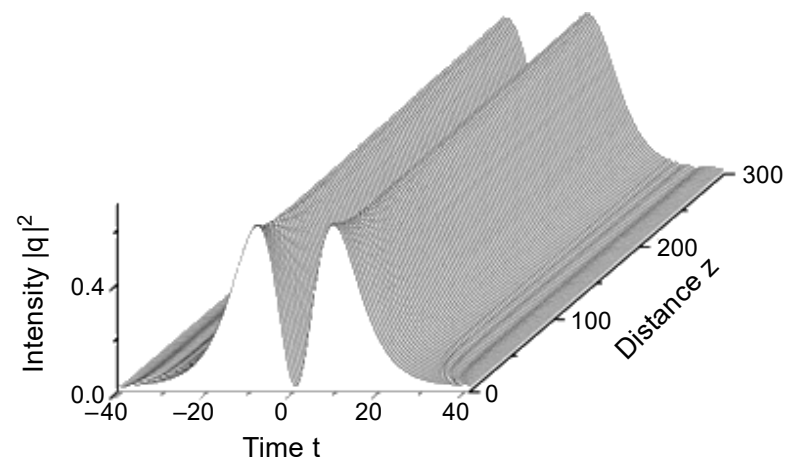

Fig. 1. The evolution plot of the M-type combined wave at a distance of 300 dispersion lengths.

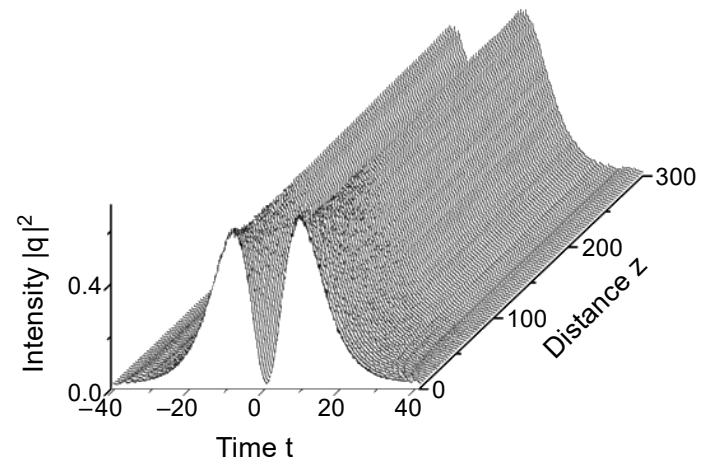

Fig. 2. The evolution plot of the M-type combined wave with noise at a distance of 300 dispersion lengths.

In order to do more in-depth study transmission characteristics of the M-type combined soliton wave in the normal dispersion regime of birefringent fiber, we investigate the transmission stability when noise interference is added. Keeping the same parameters as Fig. 1 settings, the random noise of 0.03 is added in the M-type combined solitary wave, and the incident light pulse function form is as follows:

$$
\left\{\begin{array}{l}
p(0, t)=i \beta+A_{0} \tanh \left[\eta\left(t-T_{0}\right)\right] \operatorname{sech}\left[\eta\left(t-T_{0}\right)\right]+0.03 \operatorname{rand}(t) \\
q(0, t)=i \beta+A_{0} \tanh \left[\eta\left(t-T_{0}\right)\right] \operatorname{sech}\left[\eta\left(t-T_{0}\right)\right]+0.03 \operatorname{rand}(t)
\end{array}\right.
$$

where the "rand" represents all the random numbers from 0 to 1 . The M-type combined solitary wave is transmitted through 300 dispersion lengths in the normal dispersion regime of birefringent fiber, and we obtain the evolution plot of the M-type combined wave with noise whose maximal value is 0.03 , as shown in Fig. 2.

Keeping the same parameter settings as Fig. 1, we investigate the transmission properties of the M-type combined solitary wave with the small perturbation of amplitude and phase position, and the forms of incident light pulse respectively are: 


$$
\begin{aligned}
& \left\{\begin{array}{l}
p(0, t)=0.95\left\{i \beta+A_{0} \tanh \left[\eta\left(t-T_{0}\right)\right] \operatorname{sech}\left[\eta\left(t-T_{0}\right)\right]\right\} \\
q(0, t)=0.95\left\{i \beta+A_{0} \tanh \left[\eta\left(t-T_{0}\right)\right] \operatorname{sech}\left[\eta\left(t-T_{0}\right)\right]\right\}
\end{array}\right. \\
& \left\{\begin{array}{l}
p(0, t)=\left\{i \beta+A_{0} \tanh \left[\eta\left(t-T_{0}\right)\right] \operatorname{sech}\left[\eta\left(t-T_{0}\right)\right]\right\} \exp (i \alpha) \\
q(0, t)=\left\{i \beta+A_{0} \tanh \left[\eta\left(t-T_{0}\right)\right] \operatorname{sech}\left[\eta\left(t-T_{0}\right)\right]\right\} \exp (i \alpha)
\end{array}\right.
\end{aligned}
$$

The evolution plots are shown in Figs. 3 and 4, and $\alpha=0.05$.

Not only the M-type combined wave can be stably transmitted in the normal dispersion regime, but also it shows good transmission stability in the anomalous disper-

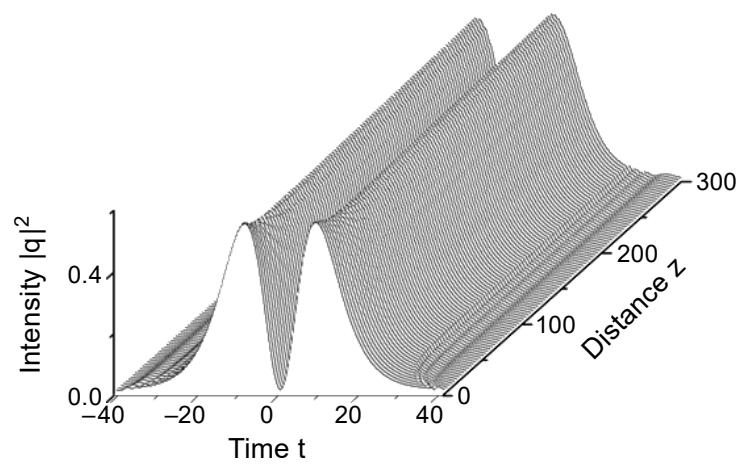

Fig. 3. The evolution plot of the M-type combined wave with amplitude perturbation at a distance of 300 dispersion lengths.

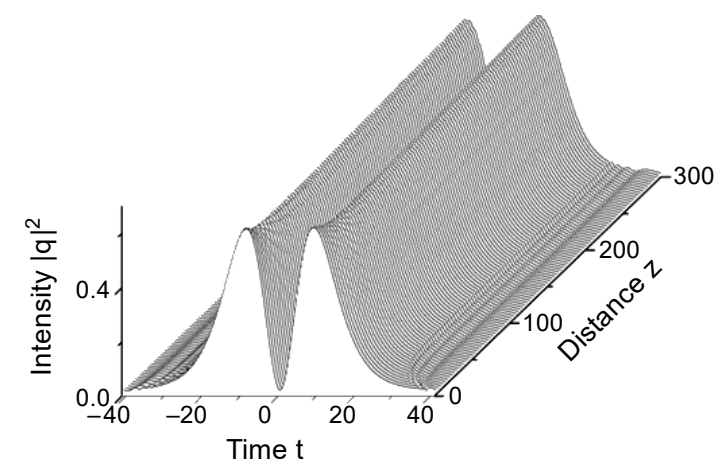

Fig. 4. The evolution plot of the M-type combined wave with phase perturbation at a distance of 300 dispersion lengths. 
sion zone by a large numbers of numerical simulations. The parameters of pulse and optical fiber are as follows: $A_{0}=1.55, \beta=0.12, T_{0}=0, \eta=0.1, \delta=0.001, g=0.0001$, $\sigma=0.2, \mu=-0.08, a=0.1, b_{20}=-0.4, b_{30}=0.08, \gamma_{30}=0.1, a_{30}=0.03, a_{40}=-0.02$, and $a_{50}=0.002$.

The evolution plot of the M-type combined wave in the anomalous dispersion regime of birefringent fiber is shown in Fig. 5. The M-type combined wave can be stably transmitted through 300 dispersion lengths in the anomalous dispersion zone, except for there is a self-adjusting process at the beginning of the transmission.

In order to study the transmission characteristics of the M-type combined soliton wave in the anomalous dispersion regime of birefringent fiber better, keeping the same parameter settings as Fig. 5, we investigate the transmission stability of the M-type combined soliton with the random noise of 0.03 , and the evolution plot is shown in Fig. 6. From the picture, we can see that the small perturbation of noise has smaller influence in the early transmission of M-type combined soliton wave in the anomalous dispersion zone, and the combined soliton wave can be stably transmitted with the increasing of transmission distance. And we find that there are slightly different influences between the two peaks of the M-type wave.

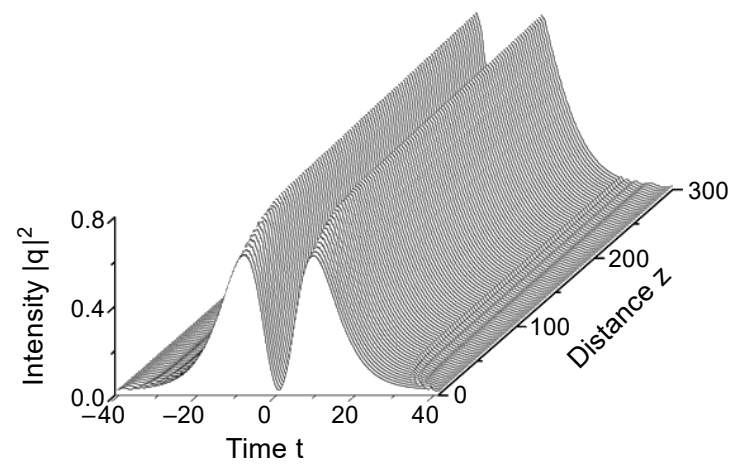

Fig. 5. The evolution plot of the M-type combined wave at a distance of 300 dispersion lengths.

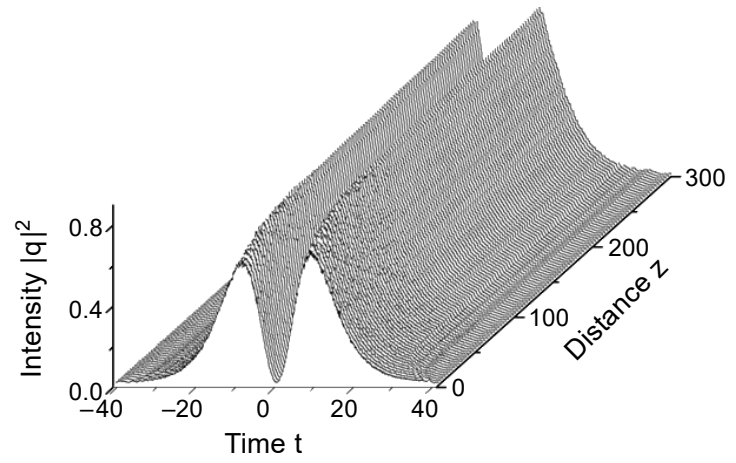

Fig. 6. The evolution plot of the M-type combined wave with noise at a distance of 300 dispersion lengths. 


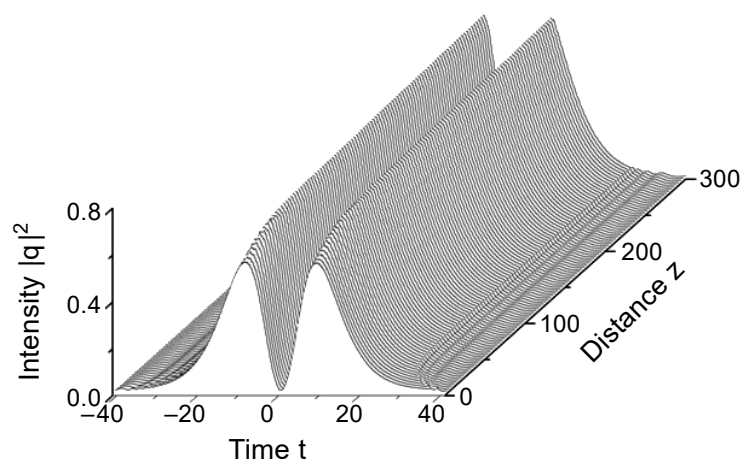

Fig. 7. The evolution plot of the M-type combined wave with amplitude perturbation at a distance of 300 dispersion lengths.

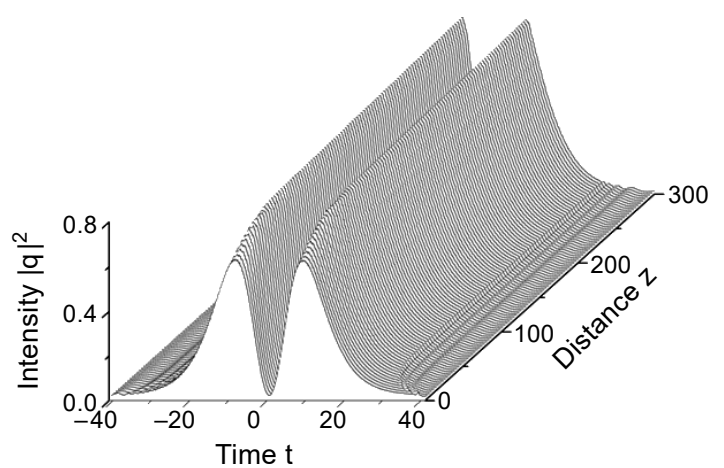

Fig. 8. The evolution plot of the M-type combined wave with phase perturbation at a distance of 300 dispersion lengths.

Keeping the same parameter settings as Fig. 5, we also investigate the transmission stability of the M-type combined wave in the anomalous dispersion regime of birefringence fiber with the small perturbation of amplitude and phase position, and the transmission evolution plots are shown in Figs. 7 and 8, respectively. In the anomalous dispersion regime, the M-type combined soliton wave can be stably transmitted with the small perturbation of amplitude and phase position.

\section{Conclusion}

The transmission properties of an optical pulse which passes through a birefringent fiber are analyzed in this paper, and the heterogeneity of this birefringent fiber is considered. We numerical simulate the transmission characteristics of the M-type combined soliton wave. The results show that the M-type combined soliton wave can be stably transmitted in the normal dispersion and anomalous dispersion regime of the 
birefringent fiber under the balance of the dispersion, self-frequency shift, self-steepening, five-order nonlinear and gain (loss) effect. And it can even be stably transmitted under the condition of limited interference. These results provide a certain reference for optical instrument research, and the development research of optical communication system.

Acknowledgments - This work was funded by National Natural Science Foundation of China (Grants Nos. 61575115 and 61378039).

\section{References}

[1] Agrawal G.P., Nonlinear Fiber Optics, Academic Press, New York, 1995, pp. 188-208.

[2] Hasegawa A., Kodama Y., Solitons in Optical Communications, Oxford University Press, Oxford, 1995, pp. 153-161.

[3] Kао K.C., Носкнам G.A., Dielectric-fibre surface waveguides for optical frequencies, Electromagnetic Wave Theory 113(3), 1966, pp. 441-444.

[4] Kodama Y., Hasegawa A., Amplification and reshaping of optical solitons in a glass fiber-II, Optics Letters 7(7), 1982, pp. 339-341, DOI: 10.1364/OL.7.000339.

[5] Mollenauer L.F., Stolen R.H., Gordon J.P., Experimental observation of picosecond pulse narrowing and solitons in optical fibers, Physical Review Letters 45(13), 1980, pp. 1095-1098, DOI: $10.1103 /$ PhysRevLett.45.1095.

[6] Agrawal G.P., Nonlinear Fiber Optics, 3rd Ed., Publishing House of Electronic Industry, Beijing, 2002, pp. 10-12, 129-156.

[7] Rashleigh S., Origins and control of polarization effects in single-mode fibers, Journal of Lightwave Technology 1(2), 1983, pp. 312-331, DOI: 10.1109/JLT.1983.1072121.

[8] Hasegawa A., Kodama Y., Solitons in Optical Communications, Oxford University Press, Oxford, 1995, pp. 11-12.

[9] Christodoulides D.N., Joseph R.I., Discrete self-focusing in nonlinear arrays of coupled waveguides, Optics Letters 13(9), 1988, pp. 794-796, DOI: 10.1364/OL.13.000794.

[10]ZaKharov V.F., SHABAT A.B., Exact theory of two-dimensional self-focusing and one-dimensional self-modulation of waves in nonlinear media, Journal of Experimental and Theoretical Physics JETP 34(1), 1972, pp. 62-69.

[11] Li Z., Li L., Tian H., Zhou G., New types of solitary wave solutions for the higher order nonlinear Schrödinger equation, Physical Review Letters 84(18), 2000, pp. 4096-4099, DOI: 10.1103/Phys $\underline{\text { RevLett.84.4096. }}$

[12] Fang F., XIaO Y., Stability of chirped bright and dark soliton-like solutions of the cubic complex Ginzburg-Landau equation with variable coefficients, Optics Communications 268(2), 2006, pp. 305-310, DOI: 10.1016/j.optcom.2006.07.014.

[13] Sun W.-R., Tian B., Wang Y.-F., Zhen H.-L., Dark single- and double-hump vector solitons of the coupled higher-order nonlinear Schrödinger equations in the birefringent or two-mode fibers, Optics Communications 335, 2015, pp. 237-244, DOI: 10.1016/j.optcom.2014.09.007.

[14] Assanto G., MacNeil J.M.L., Smyth N.F., Diffraction-induced instability of coupled dark solitary waves, Optics Letters 40(8), 2015, pp. 1771-1774, DOI: 10.1364/OL.40.001771.

[15] Kong Y., Spatiotemporal soliton solution to generalized nonlinear Schrödinger equation with a parabolic potential in Kerr media, Optics Communications 371, 2016, pp. 27-33, DOI: 10.1016/j.optcom. 2016.03.004. 
[16] Arshad M., Seadawy A.R., Lu D., Exact bright-dark solitary wave solutions of the higher-order cubic-quintic nonlinear Schrödinger equation and its stability, Optik 138, 2017, pp. 40-49, DOI: 10.1016/j.ijleo.2017.03.005.

[17] Messouber A., Triki H., Azzouzi F., Zhou Q., Biswas A., Moshokon S.P., Belic M., Propagation properties of dipole-managed solitons through an inhomogeneous cubic-quintic-septic medium, Optics Communications 425, 2018, pp. 64-70, DOI: 10.1016/j.optcom.2018.04.051.

Received April 25, 2019

in revised July 4, 2019 